\title{
Canonical quantization and expanding metrics
}

\author{
Eleonora Alfinito* and Giuseppe Vitiello ${ }^{\dagger}$ \\ Dipartimento di Fisica, Università di Salerno, 84100 \\ and INFN Gruppo Collegato di Salerno
}

\begin{abstract}
The canonical formalism for expanding metrics scenarios is presented. Nonunitary time evolution implied by expanding geometry is described as a trajectory over unitarily inequivalent representations at different times of the canonical commutation relations. Thermal properties of inflating Universe are also discussed.
\end{abstract}

\section{PACS: 04.60.d, 98.80.c}

In this paper we study the quantum field theory (QFT) formalism for non-unitary time evolution implied by inflationary models and in general by expanding metrics scenarios. As well known, non-unitary time evolution cannot be handled in the canonical formalism and for this reason many semi-classical techniques are currently used; the problem of the canonical quantization of inflationary time evolution is thus still at an unsatisfactory stage. In this paper we show that the operator formalism for the canonical quantization of time evolution in expanding geometry can be formulated provided the full set of unitarily inequivalent representations of the canonical commutation relations are considered.

Let us start our discussion, which will be limited to gravitational wave modes, by denoting, as customary, the flat time-dependent metrics by $g_{\mu \nu}(t)=g_{\mu \nu}^{0}(t)+h_{\mu \nu}(t)$. As well known, use of the De Donder gauge condition $\partial_{\mu} h_{\mu \nu}=0$ and the Einstein equations give

$$
h_{\mu \nu}=0 \text {. }
$$

The field $h_{\mu \nu}$ may be then decomposed into harmonic modes $u_{k}$ obeying the equation:

$$
\ddot{u}_{k}(t)+H \dot{u}_{k}(t)+\omega_{k}^{2}(t) u_{k}(t)=0
$$

with

$$
\omega_{k}^{2}(t)=\frac{k^{2} c^{2}}{a^{2}(t)}, \quad a(t)=a_{0} e^{\frac{1}{3} H t} .
$$

\footnotetext{
*E-mail:alfinito@pcvico.csied.unisa.it

${ }^{\dagger}$ E-mail:vitiello@pcvico.csied.unisa.it
} 
$H$ is the Hubble constant. In the Minkowski space-time $\omega_{k}$ is constant in time, but when the Universe expands, $\omega_{k}$ is time-dependent: $\omega_{k}=\omega_{k}(t)$.

In the following, where not strictly necessary, we will omit the $k$-index, remembering that each equation is written down for fixed $k$.

The first order derivative term $H u_{k}$ in eq.(2) is generally incorporated into the frequency term by using the conformal time variable $\eta$ [1, 2, 3]; such a computational strategy is very useful in the phenomenological approach, however our purpose in this paper is to illustrate the subtleties of the canonical quantization for non-unitary time evolution and therefore we must explicitly take care of the inflation term in eq.(2). In this way the full structure of the state space will be revealed.

Of course, it is the term $H \dot{u}_{k}$ in (2) which makes impossible to proceed to canonical quantization (as a matter of fact, it is not even possible to define a canonical conjugate momentum to the $u$ variable in (2)). However, as we will show in the following, the double oscillator system

$$
\begin{aligned}
& \ddot{u}+H \dot{u}+\omega^{2}(t) u=0, \\
& \ddot{v}-H \dot{v}+\omega^{2}(t) v=0,
\end{aligned}
$$

does admit a canonical quantization procedure, provided one works in the QFT framework [4, 5, 6]. Note that in the same way as the $u$ oscillator describes the expanding (inflating) metrics, the oscillator $v$ can be associated to the "contracting" ("deflating") metrics (in this sense we might speak of a "double Universe" [7, 8]): The $u-v$ system is a non-inflating (and non-deflating) system. This is why it is now possible to set up the canonical quantization scheme.

In order to better understand the need to double the degrees of freedom, it is useful to observe that $v=u e^{H t}$ is solution of (5) and that by setting $u(t)=\frac{1}{\sqrt{2}} r(t) e^{\frac{-H t}{2}}$ and $v(t)=\frac{1}{\sqrt{2}} r(t) e^{\frac{H t}{2}}$ the system of equations (团) and (5) is equivalent to the single parametric oscillator $r(t)$ (see also [9]): $\ddot{r}+\Omega^{2}(t) r=0$.

The physical reason to double the degrees of freedom relies thus in the fact that one must work with closed systems as required, indeed, by the canonical quantization formalism.

We stress that the doubling of the degrees of freedom is intrinsic to the Bogolubov transformations (see below), so that one deals with a doubled system anytime one works with such transformations. For this reason all the "mixed modes" formalisms (since Parker's work [10] ) necessarily involve the algebraic structure of the doubling of the modes.

It turns out to be convenient to introduce the canonical transformations

$$
u(t)=\frac{U(t)+V(t)}{\sqrt{2}}, \quad v(t)=\frac{U(t)-V(t)}{\sqrt{2}} .
$$

We are thus dealing with the decomposition of the parametric oscillator $r(t)$ on the hyperbolic plane (i.e. in the pseudo-Euclidean metrics): $r^{2}(t)=U^{2}(t)-V^{2}(t)$. The Hamiltonian for our coupled oscillator system is [6, 7]:

$$
\mathcal{H}=\frac{1}{2} p_{U}^{2}+\frac{1}{2} \Omega^{2}(t) U^{2}-\frac{1}{2} p_{V}^{2}-\frac{1}{2} \Omega^{2}(t) V^{2}-\Gamma\left(p_{U} V+p_{V} U\right) .
$$


with $\Gamma \equiv \frac{H}{2}$ and $\Omega(t) \equiv\left[\left(\omega^{2}(t)-\frac{H^{2}}{4}\right)\right]^{\frac{1}{2}}$, which we assume to be real (for any $k$ and any $t$ ) in order to avoid over-damped regime; i.e. we assume $\Omega^{2}(t) \geq 0$. As we will show later on, this condition turns out to act as a cut-off on $k$.

Now it is possible to introduce the annihilation operators:

$$
A=\frac{1}{\sqrt{2}}\left(\frac{p_{U}}{\sqrt{\hbar \omega_{0}}}-i U \sqrt{\frac{\omega_{0}}{\hbar}}\right), \quad B=\frac{1}{\sqrt{2}}\left(\frac{p_{V}}{\sqrt{\hbar \omega_{0}}}-i V \sqrt{\frac{\omega_{0}}{\hbar}}\right)
$$

and the corresponding creation operators with usual commutation relations.

Then it can be shown [6] that the vacuum state $\mid 0>$ is unstable:

$$
<0(t) \mid 0>\propto \exp (-t \Gamma) \rightarrow 0 \text { for large } t
$$

i.e. time evolution brings "out" of the initial-time Hilbert space for large $t$. This is not acceptable in quantum mechanics since there the Von Neumann theorem states that all the representations of the canonical commutation relations are unitarily equivalent and therefore, as already remarked above, there is no room in quantum mechanics for non-unitary time evolution as the one in (9). On the contrary, in QFT there exist infinitely many unitarily inequivalent representations and this leads to us to study our problem in the framework of QFT.

To set up the formalism in QFT we have to consider the infinite volume limit; however, as customary, we will work at finite volume and at the end of the computations we take the limit $V \rightarrow \infty$. The QFT Hamiltonian is

$$
\begin{gathered}
\mathcal{H}=\mathcal{H}_{0}+\mathcal{H}_{I_{1}}+\mathcal{H}_{I_{2}} \\
\mathcal{H}_{0}=\sum_{k} \frac{1}{2} \hbar \Omega_{0, k}(t)\left(A_{k}^{\dagger} A_{k}-B_{k}^{\dagger} B_{k}\right) \equiv \sum_{k} \hbar \Omega_{0, k}(t) \mathcal{C}_{k} \\
\mathcal{H}_{I_{1}}=-\sum_{k} \frac{1}{4} \hbar \Omega_{1, k}(t)\left[\left(A_{k}^{2}+A_{k}^{\dagger}\right)-\left(B_{k}^{2}+B_{k}^{\dagger}\right)\right] \equiv-\sum_{k} \hbar \Omega_{1, k}(t) K_{1, k} \\
\mathcal{H}_{I_{2}}=i \sum_{k} \Gamma_{k} \hbar\left(A_{k}^{\dagger} B_{k}^{\dagger}-A_{k} B_{k}\right) \equiv i \sum_{k} \hbar \Gamma_{k}\left(J_{+, k}-J_{-, k}\right)
\end{gathered}
$$

with $\Omega_{0,1, k}=\omega_{0}\left(\frac{\Omega_{k}^{2}(t)}{\omega_{0}^{2}} \pm 1\right) \quad$ and

$$
\left[A_{k}, A_{k^{\prime}}^{\dagger}\right]=\delta_{k, k^{\prime}}=\left[B_{k}, B_{k^{\prime}}^{\dagger}\right], \quad\left[A_{k}, B_{k^{\prime}}\right]=0=\left[A_{k}^{\dagger}, B_{k^{\prime}}^{\dagger}\right]
$$

The group structure underlying the Hamiltonian (10) is the one of $\mathrm{SU}(1,1)$; in fact, the operators $K_{0, k} \equiv \mathcal{C}_{k}, K_{1, k}$ and $K_{2, k}=i \frac{1}{4}\left[\left(A_{k}^{2}-A_{k}^{\dagger^{2}}\right)+\left(B_{k}{ }^{2}-B_{k}^{\dagger^{2}}\right)\right]$ close the $\mathrm{su}(1,1)$ algebra:

$$
\left[K_{1, k}, K_{2, k}\right]=-i K_{0, k}, \quad\left[K_{2, k}, K_{0, k}\right]=i K_{1, k}, \quad\left[K_{0, k}, K_{1, k}\right]=i K_{2, k}
$$


Similarly, the operators $J_{+, k}=A_{k}^{\dagger} B_{k}^{\dagger}, J_{-, k}=A_{k} B_{k}, J_{0, k}=\frac{1}{2}\left(A_{k}^{\dagger} A_{k}+B_{k}^{\dagger} B_{k}+1\right)$ close the $\mathrm{su}(1,1)$ algebra

$$
\left[J_{+, k}, J_{-, k}\right]=-2 J_{3, k} \quad, \quad\left[J_{3, k}, J_{ \pm, k}\right]= \pm J_{ \pm, k} .
$$

$\mathcal{C}_{k}$ and $J_{2, k}$ are proportional to the Casimir operators for the algebra generated by the $J$ 's operators and the $K$ 's operators, respectively.

We remark that

$$
\left[\mathcal{H}_{0}, \mathcal{H}_{I_{2}}\right]=0=\left[\mathcal{H}_{I_{1}}, \mathcal{H}_{I_{2}}\right],
$$

which guarantees that the minus sign appearing in $\mathcal{H}_{0}$ is not harmful, i.e., once one starts with a positive definite Hamiltonian it remains lower bounded under time evolution.

By using the transformation $\mathcal{H} \rightarrow \mathcal{H}^{\prime} \equiv S(\theta)^{-1} \mathcal{H} S(\theta)$ with $S(\theta) \equiv \prod_{k} e^{-i \theta_{k}(t) K_{2, k}}$ and

$$
\tanh \theta_{k}(t)=-\frac{\Omega_{1, k}(t)}{\Omega_{0, k}(t)}
$$

at any $t$ for any given $k$ we can "rotate away" [11] $\mathcal{H}_{I_{1}}$ :

$$
\mathcal{H}^{\prime} \equiv S(\theta)^{-1} \mathcal{H} S(\theta)=\mathcal{H}_{0}^{\prime}+\mathcal{H}_{I_{2}} .
$$

Notice that

$$
\mathcal{H}^{\prime}{ }_{0}=\sum_{k} \hbar \Omega_{k}(t)\left(A_{k}^{\dagger} A_{k}-B_{k}^{\dagger} B_{k}\right)
$$

and $\left[\mathcal{H}_{0}^{\prime}, \mathcal{H}_{I_{2}}\right]=0$. Also, in a consistent way, the modulus of $\frac{\Omega_{1}(t)}{\Omega_{0}(t)}$ is less or equal to 1 for any $t$ for any given $k$. Later we will comment more on this point.

When the initial state, say at arbitrary initial time $t_{0},\left(t_{0}=0, \theta_{k}(0) \equiv \theta_{k}\right.$ for sake of simplicity) is the vacuum $\mid 0>$ for $\mathcal{H}^{\prime}{ }_{0}$, with $A_{k}\left|0>=0=B_{k}\right| 0>$, the state $|0(\theta)>=S(\theta)| 0>$ is the zero energy eigenstate (the vacuum) of $\mathcal{H}_{0}+\mathcal{H}_{I_{1}}$ at $t_{0}$ :

$$
\left.\left(\mathcal{H}_{0}+\mathcal{H}_{I_{1}}\right)\right|_{t_{0}}\left|0(\theta)>=S(\theta) \mathcal{H}_{0}^{\prime}\right| 0>=0 .
$$

We observe that the operators $A_{k}$ and $B_{k}$ transform under $\exp \left(-i \theta_{k} K_{2, k}\right)$ as

$$
\begin{aligned}
& A_{k} \mapsto A_{k}(\theta)=e^{-i \theta_{k} K_{2, k}} A_{k} e^{i \theta_{k} K_{2, k}}=A_{k} \cosh \left(\frac{1}{2} \theta_{k}\right)+A_{k}^{\dagger} \sinh \left(\frac{1}{2} \theta_{k}\right), \\
& B_{k} \mapsto B_{k}(\theta)=e^{-i \theta_{k} K_{2, k}} B_{k} e^{i \theta_{k} K_{2, k}}=B_{k} \cosh \left(\frac{1}{2} \theta_{k}\right)+B_{k}^{\dagger} \sinh \left(\frac{1}{2} \theta_{k}\right) .
\end{aligned}
$$

These transformations are nothing else than the squeezing transformations and preserve the commutation relations (14). One has $A_{k}(\theta)\left|0(\theta)>=0=B_{k}(\theta)\right| 0(\theta)>$.

The state $\mid 0(\theta)>$ is thus the squeezed vacuum (at this level actually it is not, strictly speaking, a squeezed state since squeezed states are obtained by applying the squeezing generator to a (Glauber-type) coherent state). Thus we recover the squeezing phenomenon in inflating model discussed elsewhere [1, 2, 3, 12]. 
Using the commutativity of $J_{2}$ with $K_{2}$, the $t$-evolution of the squeezed vacuum $\mid 0(\theta)>$ is obtained as (at finite volume $V$ ):

$$
\left|0(\theta, t)>=\prod_{k} \frac{1}{\cosh \left(\Gamma_{k} t\right)} \exp \left(\tanh \left(\Gamma_{k} t\right) J_{k,+}(\theta)\right)\right| 0(\theta)>
$$

with $J_{k,+}(\theta)=A_{k}^{\dagger}(\theta) B_{k}^{\dagger}(\theta)$. We have $A_{k}(\theta, t)\left|0(\theta, t)>=0=B_{k}(\theta, t)\right| 0(\theta, t)>$ with

$$
\begin{aligned}
& A_{k}(\theta) \mapsto A_{k}(\theta, t)=e^{-i \frac{t}{\hbar} \mathcal{H}_{I_{2}}} A_{k}(\theta) e^{i \frac{t}{\hbar} \mathcal{H}_{I_{2}}}=A_{k}(\theta) \cosh \left(\Gamma_{k} t\right)-B_{k}(\theta)^{\dagger} \sinh \left(\Gamma_{k} t\right) \\
& B_{k}(\theta) \mapsto B_{k}(\theta, t)=e^{-i \frac{t}{\hbar} \mathcal{H}_{I_{2}}} B_{k}(\theta) e^{i \frac{t}{\hbar} \mathcal{H}_{I_{2}}}=-A_{k}(\theta)^{\dagger} \sinh \left(\Gamma_{k} t\right)+B_{k}(\theta) \cosh \left(\Gamma_{k} t\right)
\end{aligned}
$$

Notice that these are the time-dependent, canonical Bogolubov transformations.

The state $\mid 0(\theta, t)>$ is a normalized state, $<0(\theta, t) \mid 0(\theta, t)>=1 \quad \forall t$, and is a $s u(1,1)$ generalized coherent state. Provided $\sum_{k} \Gamma_{k}>0$, non-unitary time evolution is now expressed by (cf. (9)):

$$
<0(\theta, t) \mid 0(\theta)>\propto \exp \left(-t \sum_{k} \Gamma_{k}\right) \rightarrow 0 \text { for large } t \text {. }
$$

Use of the customary continuous limit relation $\sum_{k} \mapsto \frac{V}{(2 \pi)^{3}} \int d^{3} k$, for $\int d^{3} k \ln \cosh \left(\Gamma_{k} t\right)$ finite and positive, gives in the infinite volume limit

$$
\begin{gathered}
<0(\theta, t) \mid 0(\theta)>\underset{V \rightarrow \infty}{\longrightarrow} 0 \quad \forall t \quad, \\
<0(\theta, t) \mid 0\left(\theta^{\prime}, t^{\prime}\right)>\underset{V \rightarrow \infty}{\longrightarrow} 0 \text { with } \theta^{\prime} \equiv \theta\left(t_{0}^{\prime}\right), \quad \forall t, t^{\prime}, t_{0}^{\prime}, \quad t \neq t^{\prime} .
\end{gathered}
$$

Eqs. (28) and (29) show that in the infinite volume limit the vacua at $t$ and at $t^{\prime}$, for any $t$ and $t^{\prime}$, are orthogonal states and thus the corresponding Hilbert spaces are unitarily inequivalent spaces. This means that the set of states of the system splits into unitarily inequivalent representations $\{\mid 0(\theta, t)>\}$ labeled by $t$.

Thus, the result we have obtained is that the system in its evolution runs over a variety of representations labeled by $t$ of the canonical commutation relations which are unitarily inequivalent to each other for $t \neq t^{\prime}$ in the infinite-volume limit: the nonunitary character of time evolution implied by expanding geometry is thus recovered, in a consistent scheme, in the unitary inequivalence among representations at different times in the infinite volume limit.

The number of modes of type $A_{k}(\theta)$ in the state $|0(\theta, t)\rangle$ is given, at each instant $t$ by

$$
n_{A_{k}}(t) \equiv<0(\theta, t)\left|A_{k}^{\dagger}(\theta) A_{k}(\theta)\right| 0(\theta, t)>=\sinh ^{2}\left(\Gamma_{k} t\right) \quad,
$$

and similarly for the modes of type $B_{k}(\theta)$.

We also observe that the commutativity of $\mathcal{C}$ (i.e. $K_{0}$ ) with $\mathcal{H}_{I_{2}}$ (i.e. $J_{2}$ ) ensures that the number $\left(n_{A_{k}}-n_{B_{k}}\right)$ is a constant of motion for any $k$ and any $\theta$. Moreover, one can show [6, 14] that the creation of a mode $A_{k}(\theta)$ is equivalent to the destruction 
of a mode $B_{k}(\theta)$ and vice-versa. This means that the $B_{k}(\theta)$ modes can be interpreted as the holes for the modes $A_{k}(\theta)$ : the $B$-system can be considered as the sink where the energy dissipated by the $A$-system flows.

Notice that in the continuum limit, as well known, the $A_{k}$ (and $B_{k}$ ) operators are not well defined on vectors in the Fock space; for instance, since $\left[A_{k}, A_{k^{\prime}}^{\dagger}\right]=$ $\delta\left(k-k^{\prime}\right), \quad\left|A_{k}>\equiv A_{k}^{\dagger}\right| 0>$ is not a normalizable vector: $\left\langle A_{k} \mid A_{k}\right\rangle=\delta(0) \quad$ which is infinity. As customary one must then introduce wave-packet (smeared out) operators $A_{f}=\frac{1}{(2 \pi)^{3 / 2}} \int d^{3} k A_{k} f(k)$, with spatial distribution described by square-integrable (orthonormal) functions $f(x)$. The commutators are

$$
\left[A_{f}, A_{g}^{\dagger}\right]=(f, g)=\left[B_{f}, B_{g}^{\dagger}\right], \quad\left[A_{f}, B_{g}\right]=0, \quad\left[A_{f}^{\dagger}, B_{g}^{\dagger}\right]=0,
$$

with $(f, g)$ denoting the scalar product between $f$ and $g$. Now $<A_{f} \mid A_{f}>=1$ and the $A_{f}$ 's are well defined operators in the Fock space where observables have to be realized. The $A_{f}$ number operator is then

$$
n_{A_{f}}(t)=<0(\theta, t)\left|A_{f}^{\dagger}(\theta) A_{f}(\theta)\right| 0(\theta, t)>=\frac{1}{(2 \pi)^{3}} \int d^{3} k \sinh ^{2}\left(\Gamma_{k} t\right)|f(k)|^{2},
$$

and similarly for the modes of type $B_{f}(\theta)$ (cf. with eq. (30)). We can set $n_{A_{f}}(t) \equiv$ $\sinh ^{2}(\Gamma t)$ and Eq. (32) then specifies the relation between the $\Gamma_{k}$ 's and $\Gamma \equiv H / 2$.

The structure of $\mid 0(\theta, t)>$ naturally leads us to recognize its thermal properties. The vacuum state $\mid 0(\theta, t)>$ can be written as

$$
\left|0(\theta, t)>=\exp \left(-\frac{1}{2} \mathcal{S}_{A(\theta)}\right)\right| \mathcal{I}(\theta)>=\exp \left(-\frac{1}{2} \mathcal{S}_{B(\theta)}\right) \mid \mathcal{I}(\theta)>
$$

where $\left|\mathcal{I}(\theta)>\equiv \exp \left(\sum_{k} A_{k}^{\dagger}(\theta) B_{k}^{\dagger}(\theta)\right)\right| 0(\theta)>$ is the invariant (not normalizable) vector [14] and

$$
\mathcal{S}_{A(\theta)} \equiv-\sum_{k}\left\{A_{k}^{\dagger}(\theta) A_{k}(\theta) \ln \sinh ^{2}\left(\Gamma_{k} t\right)-A_{k}(\theta) A_{k}^{\dagger}(\theta) \ln \cosh ^{2}\left(\Gamma_{k} t\right)\right\} .
$$

$\mathcal{S}_{B(\theta)}$ has the same expression with $B_{k}(\theta)$ and $B_{k}^{\dagger}(\theta)$ replacing $A_{k}(\theta)$ and $A_{k}^{\dagger}(\theta)$, respectively. In the following we shall simply write $\mathcal{S}(\theta)$ for either $\mathcal{S}_{A(\theta)}$ or $\mathcal{S}_{B(\theta)}$. $\mathcal{S}(\theta)$ is recognized to be the entropy [6, 14.

Since the $B$-particles are the holes for the $A$-particles, $\mathcal{S}_{A(\theta)}-\mathcal{S}_{B(\theta)}$ is in fact the (conserved) entropy for the closed system: $\left[\mathcal{S}_{A(\theta)}-\mathcal{S}_{B(\theta)}, \mathcal{H}\right]=0$.

Eqs. (33) and (34) show that the operator dependence of $\frac{1}{2} \mathcal{S}_{A(\theta)}$ (or respectively, $\left.\frac{1}{2} \mathcal{S}_{B(\theta)}\right)$ is uniquely on the $A(B)$ variables: thus in eq. (33) time evolution is expressed solely in terms of the (sub)system $A(B)$ with the elimination of the $B(A)$ variables. This reminds us of the procedure by which one obtains the reduced density matrix by integrating out bath variables.

For the time variation of $\mid 0(\theta, t)>$ at finite volume $V$, we obtain

$$
\frac{\partial}{\partial t}\left|0(\theta, t)>=-\frac{1}{2}\left(\frac{\partial \mathcal{S}(\theta)}{\partial t}\right)\right| 0(\theta, t)>\quad \text {. }
$$


Equation (35) shows that $\frac{1}{2}\left(\frac{\partial \mathcal{S}(\theta)}{\partial t}\right)$ is the generator of time-translations, namely time evolution is controlled by the entropy variations. This correctly reflects the irreversibility of time evolution characteristic of expanding metrics. Expanding geometry implies in fact the choice of a privileged direction in time evolution (time arrow) with a consequent breaking of time-reversal invariance.

Let us now consider the $A$-modes alone and introduce the functional (free energy)

$$
F_{A} \equiv<0(\theta, t)\left|\left(\mathcal{H}_{0, A(\theta)}^{\prime}-\frac{1}{\beta} \mathcal{S}_{A(\theta)}\right)\right| 0(\theta, t)>
$$

Here $\mathcal{H}_{0, A(\theta)}^{\prime} \equiv \sum_{k} E_{k} A_{k}^{\dagger}(\theta) A_{k}(\theta)$ and $E_{k} \equiv \hbar \Omega_{k}\left(t_{0}=0\right)-\mu$, with $\mu$ the chemical potential. The stability condition $\frac{\partial F_{A(\theta)}}{\partial \sigma_{k}}=0, \sigma_{k} \equiv \Gamma_{k} t \forall k$, assuming $\beta$ a slowly varying functions of $\mathrm{t}$, gives $\beta E_{k}=-\ln \tanh ^{2}\left(\sigma_{k}\right)$, i.e.

$$
n_{A_{k}}(t)=\sinh ^{2}\left(\Gamma_{k} t\right)=\frac{1}{\mathrm{e}^{\beta(t) E_{k}}-1},
$$

which is the Bose distribution for $A_{k}$ at time $t$ provided we assume $\beta(t)$ to represent the inverse temperature $\beta(t)=\frac{1}{k_{B} T(t)}$ at time $t$ ( $k_{B}$ denotes the Boltzmann constant). This allows us to recognize $\{\mid 0(\theta, t)>\}$ as a representation of the canonical commutation relations at finite temperature, equivalent with the Thermo Field Dynamics representation $\{\mid 0(\beta)>\}$ of Takahashi and Umezawa [14, 15, 16]. See also [7, 8].

Let us now finally comment on the reality condition for $\Omega_{k}(t)$. Our first remark is that such a condition actually excludes long wave modes, and thus acts as an intrinsic infrared cut-off; in fact, it is easy to show that $\Omega_{k}^{2}(t) \geq 0$ for any $t$ implies $k \geq k_{0} e^{\frac{H}{3} t}$, with $k_{0} \equiv \frac{H a_{0}}{2 c}$ for any $t$. We recover in this way the known feature of inflationary models by which only in the "tight coupling" phase $\left(\lambda<R_{H}\right)$ there is an oscillatory evolution [12].

As a matter of fact, besides the reality condition, we also have the condition (18) which implies that $\Omega_{k}{ }^{2}(t) \leq \omega_{0}^{2}$ for $\theta_{k} \geq 0$ and that $\Omega_{k}{ }^{2}(t) \geq \omega_{0}^{2}$ for $\theta_{k} \leq 0$. This, together with the reality condition, leads to the bounds for $k$ :

$$
\begin{aligned}
k_{0} e^{\frac{H}{3} t} & \leq k \leq \tilde{k}_{0} e^{\frac{H}{3} t} \text { at any } t \quad \text { for } \theta_{k} \geq 0 \\
k & \geq \tilde{k}_{0} e^{\frac{H}{3} t} \quad \text { at any } t \text { for } \theta_{k} \leq 0
\end{aligned}
$$

with $\tilde{k}_{0} \equiv \frac{a_{0}}{c} \sqrt{\omega_{0}^{2}+\left(\frac{H}{2}\right)^{2}}$.

On the other hand, for each given mode $k$, the frequency $\Omega_{k}$ is different from zero only in the span of time $0 \leq t \leq \mathrm{T}_{k} \equiv \frac{3}{H} \ln \frac{k}{k_{0}}$ (limiting ourselves to positive time evolution). For instance, for each $k$ we have:

$$
\Omega_{k}\left(\Lambda_{k}(t)\right)=\Omega_{k}(0) e^{-\Lambda_{k}(t)}, \quad \Lambda_{k}(t) \geq 0 \text { for any } t
$$

with

$$
e^{-2 \Lambda_{k}(t)} \equiv \frac{e^{-t \frac{H}{3}} \sinh \frac{H}{3}\left(\mathrm{~T}_{k}-t\right)}{\sinh \frac{H}{3} \mathrm{~T}_{k}}
$$


and $\Omega_{k}\left(\Lambda_{k}(0)\right)=\Omega_{k}(0)$ and $\Omega_{k}\left(\Lambda_{k}\left(\mathrm{~T}_{k}\right)\right)=0$. Modes with larger $k$ have "longer" life with reference to time $t$. Asymptotically in $k$, the limit value $\Lambda_{\text {asimpt. }}=\frac{H}{3} t$ is reached (see figs.1 and 2). In conclusion, only the modes satisfying conditions (38) and (39) are present at time $t$, being the other ones decayed (fig. 2).

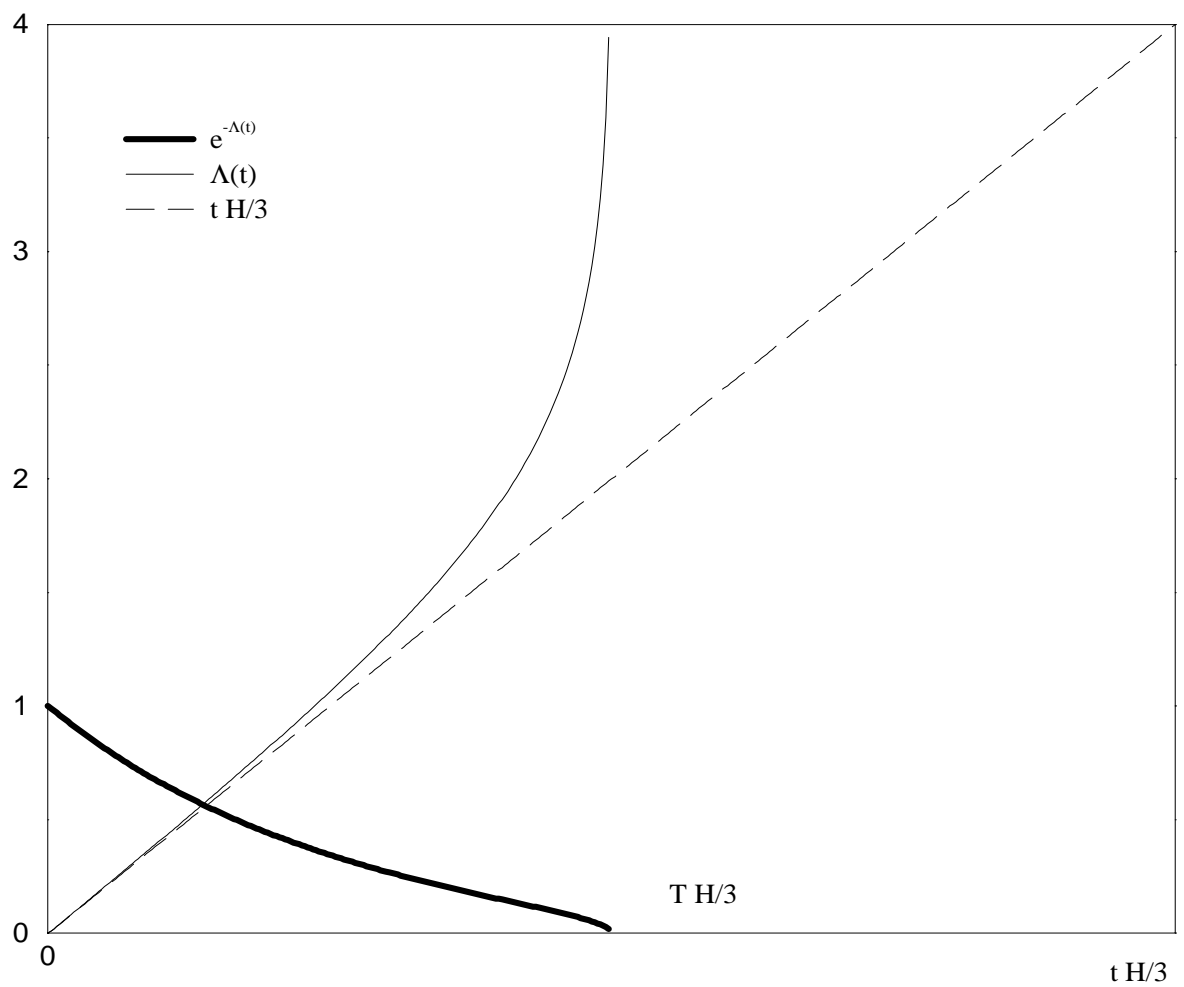

fig. 1

The condition (18) allows to write also

$$
\Omega_{k}(t)=\omega_{0} e^{-\theta_{k}(t)} .
$$

Thus we see from (40) and (42) that $\Lambda_{k}(t)=\theta_{k}(t)-\theta_{k}(0)$, i.e. defining $\Lambda_{k}(t)=$ $\gamma_{k} \tau_{k}(t)\left(\gamma_{k} \tau_{k}(0)=0, \gamma_{k} \tau\left(\mathrm{T}_{k}\right)=\infty\right)$, we have

$$
\Omega_{k}(t)=\omega_{0} e^{-\theta_{k}(0)} e^{-\gamma_{k} \tau_{k}(t)},
$$

which shows that $\tau_{k}(t)$ can be interpreted as the proper time of the $k$-mode.

It is finally interesting to remark that the number $n_{k}$ of $k$-modes condensed in the state $|0(\theta)\rangle$, given by $\sinh ^{2} \theta_{k}$, can be expressed as $n_{k} \equiv n_{+, k}+n_{-, k}$ in terms of 


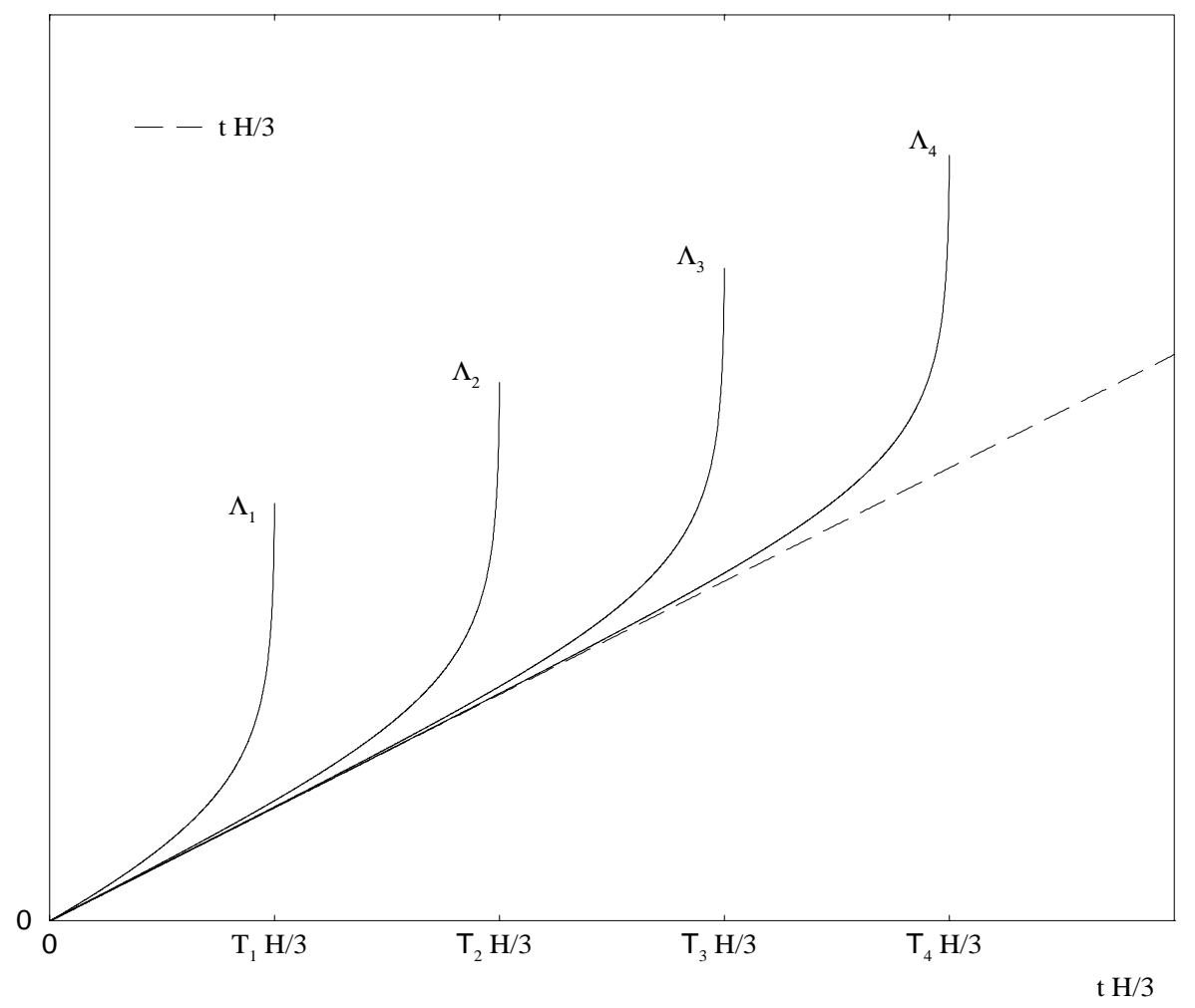

fig. 2

the $k$-modes $n_{+, k}$ and $n_{-, k}$ going forward and backwards in the proper time $\tau_{k}$, respectively:

$$
n_{ \pm, k}(t)=\frac{n_{k}(t)}{e^{\mp 2 \theta_{k}(0)} e^{\mp 2 \gamma_{k} \tau_{k}(t)}+1}
$$

Clearly, from (18) one recognizes that $n_{+, k}-n_{-, k}$ can be considered as an "order parameter" since $\tanh \theta_{k}(t)=\frac{n_{+, k}(t)-n_{-, k}(t)}{n_{k}(t)}$, and $n_{+, k}=n_{-, k}$ for any $t$ for each $k$-mode with constant frequency $\left(\theta_{k}=0\right.$ for any $\left.t\right)$.

This work has been partially supported by INFN, by MURST and by a Network of the European Science Foundation on Topological Defects. 


\section{References}

[1] L. P. Grishchuk, Zh. Eksp. Teor. Fiz. 67 (1974) 825 [ Sov. Phys. JEPT 40 (1975) 409].

[2] L. P. Grishchuk and Y. V. Sidorov, Phys. Rev. D 42 (1990) 3413 and refs. quoted therein.

[3] L. P. Grishchuk, H. A. Haus and K. Bergman, Phys. Rev. D 46 (1992) 1440.

[4] H. Feshbach and Y. Tikochinsky, Transact. N.Y. Acad. Sci. 38 (Ser. II) (1977) 44.

[5] H. Bateman, Phys. Rev. 38 (1931) 815.

[6] E. Celeghini, M. Rasetti and G. Vitiello, Ann.Phys. (N.Y.) 215 (1992) 156.

[7] E. Alfinito, R. Manka and G. Vitiello, Double Universe, hep-th/9705134.

[8] E.Alfinito and G. Vitiello, Canonical quantization for expanding Universe, in Mathematical Methods of Quantum Physics, M.V. Carpio-Bernido et al. Eds., (Gordon and Breach Sc. Publ., Amsterdam, in print).

[9] M. Blasone, E. Graziano, O. K. Pashaev and G. Vitiello, Ann. Phys. (N.Y.) 252 (1996) 115.

[10] L. Parker, Phys. Rev. D 183 (1969) 1057.

[11] A.I. Solomon, J. Math. Phys. 12 (1971) 390.

[12] A. Albrecht, P. Ferreira, M. Joyce and T. Prokopec, Phys. Rev. D (1994) 4807; A. Albrecht, Coherence and Sakharov Oscillations in the Microwave Sky, astro-ph 9612015 .

[13] A. M. Perelomov, Generalized Coherent States and their Applications (SpringerVerlag, Berlin 1986).

[14] Y. Takahashi and H. Umezawa, Collective Phenomena 2 (1975) 55.

[15] H. Umezawa, H. Matsumoto and M. Tachiki, Thermo Field Dynamics and Condensed States (North-Holland Pub. Co., Amsterdam 1982).

[16] H. Umezawa, Advanced Field Theory: Micro, Macro, and Thermal Concepts (American Institute of Physics, N.Y. 1993). 